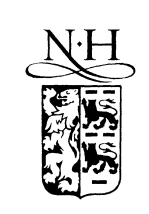

ELSEVIER

\title{
Source of non-Arrhenius average relaxation time in glass-forming liquids
}

\author{
Jeppe C. Dyre \\ Department of Mathematics and Physics (IMFUFA), Roskilde University, P.O. Box 260, DK-4000 Roskilde, Denmark
}

\begin{abstract}
A major mystery of glass-forming liquids is the non-Arrhenius temperature-dependence of the average relaxation time. This paper briefly reviews the classical phenomenological models for non-Arrhenius behavior - the free volume model and the entropy model - and critiques against these models. We then discuss a recently proposed model according to which the activation energy of the average relaxation time is determined by the work done in shoving aside the surrounding liquid to create space needed for a "flow event". In this model, which is based on the fact that intermolecular interactions are anharmonic, the non-Arrhenius temperature-dependence of the average relaxation time is a consequence of the fact that the instantaneous shear modulus increases upon cooling. (c) 1998 Elsevier Science B.V. All rights reserved.
\end{abstract}

\section{Introduction}

Apparently all supercooled liquids are able to form glasses [1-12]. The glass transition takes place when the viscosity upon cooling becomes so large that molecular motion is arrested on the time-scale of the experiment. The fascination of this phenomenon lies in the fact that chemically quite different liquids - involving ionic interactions, van der Waals forces, hydrogen bonds, covalent bonds, or even metallic bonds - have a number of common properties when cooled to become highly viscous $[3,6,7,12]$. Of particular interest here is the temperature-dependence of the average relaxation time, $\tau$. This quantity may be determined, e.g., as the inverse dielectric, mechanical or specific heat loss peak frequency. Alternatively, it may be calculated from the viscosity, $\eta$, and the instantaneous (infinite-frequency) shear modulus, $G_{\infty}$, by means of Maxwell's expression
$\tau=\frac{\eta}{G_{\infty}}$.

These definitions do not give exactly identical average relaxation times, but the difference is insignificant for the present purposes. It is widely believed $[1,3,13-25]$ that different average relaxation times are roughly identical because they basically measure the rate of "flow events": Most molecular motion in a highly viscous liquid is purely vibrational around a potential energy minimum. Only seldom does real motion take place. This happens in the form of a sudden rearrangement of molecules - a flow event - a process which is unlikely because of the large potential energy barrier to be overcome $[1,15,22]$. Kauzmann referred to flow events as "jumps of molecular units of flow between different positions of equilibrium in the liquid's quasicrystalline lattice" [1]. The molecules involved in a flow event define a "relaxing unit" [1], "cooperatively rearranging subsystem" [7] or "cooperatively rearranging region" 
[13], "quasi-independent unit" [17], "thermokinetic structure" [20], "molecular domain" [22], or "dynamically correlated domain" [23].

As the glass transition is approached, the average relaxation time becomes longer and longer. For typical cooling rates $\tau$ is of order $10^{3} \mathrm{~s}$ at the glass transition temperature, $T_{\mathrm{g}}$. From a general physical/chemical point of view, the temperaturedependence of $\tau$ is anomalous in the following sense. In only very few liquids is $\tau$ described by an Arrhenius function (examples are $\mathrm{SiO}_{2}, \mathrm{GeO}_{2}$, $\mathrm{BeF}_{2}$ or albite $\left(\mathrm{NaAlSi}_{3} \mathrm{O}_{8}\right)$ [3]). Predominantly, $\tau$ is non-Arrhenius by exhibiting an apparent activation energy [defined as $\partial \ln \tau / \partial\left(k_{B} T\right)^{-1}$ ] that increases as the temperature decreases. A measure of the departure from Arrhenius behavior is the fragility, $m$, defined as the apparent activation energy at $T=T_{\mathrm{g}}$ in units of $k_{\mathrm{B}} T_{\mathrm{g}} \ln (10)$ [26]. For an Arrhenius liquid, $m$, is about 16 ; for most viscous liquids $m$ is between 50 and 150. Liquids with large fragility are termed "fragile", liquids with fragility $\sim 16$ are termed "strong" [26]. There is a general tendency that fragile liquids have broader distributions of relaxation times than strong liquids [27,28]. This rule, however, is not without exceptions $[29,30]$.

In the discussion below we will not distinguish between the temperature-dependence of average relaxation time and of viscosity, because these two quantities are approximately proportional (in Eq. (1) the temperature-dependence of $G_{\infty}$ is insignificant). Also, no distinction is made below between activation energy and activation free energy, since this distinction was not done in many older papers in the field. We identify the temperature-dependent activation energy from the expression [25,31]

$\tau=\tau_{0} \exp \left(\frac{\Delta E(T)}{k_{\mathrm{B}} T}\right)$.

Although $\Delta E(T)$ is quite different from the apparent activation energy, experiments imply that $\Delta E(T)$ also increases as the temperature decreases.

It is not at all obvious that a general explanation for the non-Arrhenius behavior of chemically quite different viscous liquids exists, but it seems to be a reasonable first hypothesis. This paper discusses models for the non-Arrhenius average re- laxation time, models that are phenomenological in the sense that $\tau(T)$ is determined by some macroscopic property of the liquid. The most famous phenomenological models for the non-Arrhenius $\tau \mathrm{s}$ are the free volume model of Cohen, Turnbull and Grest [14,32-34] and the entropy model of Gibbs, DiMarzio and Adam [13,35]. These models and critiques against them are briefly reviewed below (see also Johari's review of phenomenological models Ref. [4]). We then discuss a recently proposed model [36,37], according to which the activation energy of a flow event mainly originates in the work done in shoving aside the surrounding liquid to create enough space for the molecules to rearrange.

\section{Early phenomenological models}

The significance of volume was emphasized long ago by Eyring and coworkers, who suggested that the viscosity of a liquid is smaller the greater the number of holes present [38]. Defining the free volume per molecule, $v_{\mathrm{f}}$, as the average volume per molecule in the liquid minus the volume of the molecule itself, in 1951 Doolittle [39] found that the viscosity of a number of simple hydrocarbon liquids may be fitted by the expression

$\eta=\eta_{0} \exp \left(\frac{C}{v_{\mathrm{f}}}\right)$

In 1959 this expression was derived by Cohen and Turnbull arguing as follows [32]. The molecules are modelled as hard spheres. A molecule is mostly confined to a cage bounded by its immediate neighbors. Occasionally, there is a fluctuation in density which opens up a hole within the cage. Molecular transport occurs only when a void having a volume larger than some critical $v^{*}$ forms. The total "free" volume may be distributed in various ways between the cages. The average relaxation time is essentially the inverse of the probability, $P$, that redistribution of free volume by chance creates a void of greater volume than $v^{*}$. Turnbull and Cohen calculated this probability by standard statistical mechanical arguments [32]. Their result is $P \propto \exp \left(-C / v_{\mathrm{f}}\right)$, leading to Eq. (3) via Eq. (1). A basic assumption in the free volume 
model is that no energy is required for redistribution of free volume. When the model is applied to real liquids, Cohen and Turnbull defined the free volume as that part of the excess volume that may be redistributed with no increase in energy [33].

In the free volume model the temperature-dependence of the average relaxation time comes from the fact that the free volume decreases with decreasing temperature. If the free volume is taken to be a linearly decreasing function of temperature, one arrives via Eq. (1) at the Vogel-Fulcher-Tammann (VFT) expression,

$\tau=\tau_{0} \exp \left(\frac{A}{T-T_{0}}\right)$.

Here $T_{0}$ is the temperature at which there is no free volume.

It is noteworthy [4] that in the approach of Cohen and Turnbull, the concept of free volume has a meaning different from that of Doolittle [39]. In Doolittle's definition, the molecular volume is obtained by extrapolating the liquid volume to zero temperature and consequently the free volume is zero only at zero temperature.

What critiques may be raised against the free volume model? The derivation of Eq. (3) may be questioned because of the primitive way in which the "entropy" (distribution) of free volume is taken into account. Moreover, despite several attempts $[14,33]$ the very concept of free volume in Cohen and Turnbull's sense seems to be ill defined operationally for general liquids. When it comes to a comparison to experiment, the VFT equation, Eq. (4), often gives a good fit to data [7,8]. However, the fit is seldom perfect; in particular, there are systematic deviations close to $T_{\mathrm{g}}$, where the average relaxation time is apparently always less temperature-dependent than predicted by Eq. (4) $[7,31,40-43]$. A direct test of the free volume model may be performed by applying pressure to the liquid, since the model predicts that the average relaxation time is solely a function of density. Indeed, $\tau$ does increase at high pressures, but quantitatively the free volume model is not confirmed [3]. A further test of the model is based on the fact that the glass transition is similar to a second-order phase transition in the sense of Ehrenfest (with continuity of first derivatives of the free energy and discontinuity of second derivatives). For the pressure-dependence of the transition temperature, the hypothesis that the average relaxation time is controlled by volume translates into the requirement that the glass transition takes place at constant volume. This requirement implies [44]

$\frac{\mathrm{d} T_{\mathrm{g}}}{\mathrm{d} p}=\frac{\Delta \kappa}{\Delta \alpha}$,

where $\Delta \kappa$ is the difference between the isothermal bulk compressibility of liquid and glass and $\Delta \alpha$ the same difference for the isobaric thermal expansion coefficient. Eq. (5) is seldom satisfied [45,46]; in fact Nieuwenhuizen has recently argued that it cannot be correct [47].

In the free volume model the glass transition occurs at a fixed volume. In the theory of Gibbs and DiMarzio from 1958 [35] the variable controlling the average relaxation time is the configurational entropy. Evaluating the partition function for a lattice model of linear polymeric chains in a mean-field approximation, Gibbs and DiMarzio found that there is a second-order phase transition at a finite temperature, $T_{\mathrm{K}}$, to a low-temperature state of zero configurational entropy. This state is a "ground state" of amorphous packing. Furthermore, Gibbs and DiMarzio argued that in the neighborhood of $T_{\mathrm{K}}$ the energy barrier restricting transitions between different molecular configurations is very large, because "the few states that could conceivably occur close to $T_{\mathrm{K}}$ are widely separated in phase space, so proceeding from one to another involves a considerable change in the topology of molecular entanglements". In this picture, the very equilibrium properties of a supercooled liquid give rise to kinetic sluggishness which prevents the equilibrium second order phase transition from being reached in finite time.

These ideas were quantified in 1965 by Adam and Gibbs [13]. They argued that the size of cooperatively rearranging regions, defined as "the smallest regions that can undergo a transition to a new configuration without a requisite simultaneous configurational change on and outside its boundary", diverges as the configurational entropy goes to zero. The region size is estimated by requiring that at least two different configurational 
states should reside in a region, leading to a size inversely proportional to the configurational entropy, $S_{\mathrm{c}}$. Assuming the energy barrier to be overcome is proportional to region size, Adam and Gibbs arrived at the following expression for the temperature-dependence of the average relaxation time,

$\tau=\tau_{0} \exp \left(\frac{C}{S_{\mathrm{c}} T}\right)$.

Close to $T_{\mathrm{K}}$ the denominator may be expanded to first order in $T-T_{\mathrm{K}}$ whereby Eq. (6) becomes the VFT expression Eq. (4) with

$T_{0}=T_{\mathrm{K}}$.

The entropy model resolves the Kauzmann paradox of a negative configurational entropy below $T_{\mathrm{K}}$ without just avoiding it (as Kauzmann did himself [1] by suggesting that crystallization sets in before $T_{\mathrm{K}}$ is reached). The model is in good agreement with experiment. Thus, Eq. (7) is often obeyed $[8,13,46,48]$, which is quite remarkable, given the dynamic definition of $T_{0}$ and the quite different purely thermodynamic definition of $T_{\mathrm{K}}$. In particular, systems with only small excess specific heats which imply smaller Kauzmann paradoxes and $T_{\mathrm{K}} \mathrm{s}$ close to zero, generally tend to be "strong", i.e., have VFT $T_{0}$ s close to zero [49].

The entropy model presents a beautiful scenario. Still, it may be critiqued both in regard to its relation to experiment and in regard to its internal consistency. Experimentally, there is no proof that a second order phase transition to a state of zero configurational entropy is underlying the laboratory glass transition. In many cases a simple two-level system model accounts well for excess entropy data [50]; more generally, the data may be fitted with models with only few energy levels [17]. Also, as mentioned above, the VFT expression fails close to $T_{\mathrm{g}}$, where $\tau$ is usually less temperature-dependent than predicted. Finally, it should be mentioned that the identification of excess entropy with configurational entropy rests on an assumption that the glass has the same "fast" contribution to the entropy as the crystal at the same temperature. As pointed out by Goldstein [51], this assumption is not always realistic because the glass may have significant contributions to the "fast" specific heat from anharmonic vibrations and secondary relaxations not present in the crystal.

In regard to the internal consistency of the entropy model, we first note that the mean-field solution of the lattice polymer model of Gibbs and DiMarzio is incorrect and that, in fact, the model has a positive configurational entropy at all positive temperatures $[52,53]$. Ignoring this objection and accepting the general idea of a phase transition to a state of zero configurational entropy, one may reasonably ask [31]: What is the nature of the amorphous ground state, the "ideal glass"? Since this state is unique a simple description of it would be expected; however none has been proposed. The argument of Adam and Gibbs is also not compelling. They assumed, ad hoc, that the energy barrier to be overcome is proportional to the size of the cooperatively rearranging region. Though this may seem reasonable, it does not have to be correct. More generally, approaching a zeroentropy state does not in itself imply a diverging relaxation time. There is no compelling link between dynamics and thermodynamics: in a master equation description of the dynamics many different possible forms of transition rates, leading to quite different relaxation behaviors, are consistent with the same statistical mechanics.

Instead of focussing on volume or entropy as the variable controlling the relaxation of viscous liquids, potential energy may be the relevant variable, as first suggested by Goldstein [15]. A number of authors have since taken this approach $[3,16,17,21-25,54,55]$. In the simplest energy controlled models $[16,17,21]$ the transition state of a region is taken to be temperature-independent with potential energy, $E_{0}$, leading to the following expression for the activation energy in terms of the average potential energy of one region, $\bar{E}(T)$ [25]:

$\Delta E(T)=E_{0}-\bar{E}(T)$.

Since the average potential energy decreases with decreasing temperature, the activation energy increases. Qualitatively, this effect is observed in experiment. However, to fit data for $\tau(T)$ relatively large regions are needed, implying much broader relaxation time distributions than observed [25]. Therefore, the simple picture does not work, and more involved approaches need to be taken [55]. 


\section{The shoving model}

The above discussed models all assume that relaxation depends only on the state of the region involved. A quite different approach may be taken, where the relaxation rate depends only on properties of the surrounding liquid. As starting point we take the fact that molecular interactions are anharmonic with strong short-ranged repulsions and weak long-ranged attractions. As shown by Widom [56] and Chandler et al. [57], this "van der Waals" picture explains a number of phenomena in liquids. Next, as in the free volume model, we assume that space is needed for molecules in a viscous liquid to rearrange. The idea is that, because of the harsh intermolecular repulsion, rearrangement at constant region volume is very costly, so it is more favorable for the molecules to spend some energy on shoving aside the surrounding liquid. Suppose the rearranging molecules constitute a sphere which at the transition increases its radius by $\Delta r$. Because the surrounding liquid may be regarded as an elastic solid on the short time scale of a flow event, the energy cost for expanding is for some constant $A$ equal to $A(\Delta r)^{2}$. The energy barrier to be overcome inside the sphere is some function, $f(\Delta r)$. Minimizing the total energy cost leads to $2 A \Delta r+f^{\prime}(\Delta r)=0$. The ratio between the "shoving" work and the "inner" barrier to be overcome is denoted by $\lambda$, thus given by

$\lambda=\frac{A(\Delta r)^{2}}{f(\Delta r)}=-\frac{1}{2} \frac{\mathrm{d} \ln \mathrm{f}}{\mathrm{d} \ln \Delta r}$.

Because of the strong repulsions one expects the logarithmic derivative of $f(\Delta r)$ to be numerically much larger than one, so the shoving work gives the dominant contribution to the energy barrier. Consequently, the "inner" contribution to the activation energy may be ignored.

To calculate the "shoving" work I use the fact that during a flow event the surrounding liquid behaves as an elastic isotropic solid with bulk modulus, $K_{\infty}$, and shear modulus, $G_{\infty}$. These elastic constants are known to be more temperature-dependent in viscous liquids than in simple liquids or solids (crystals or glasses). Both $K_{\infty}$ and $G_{\infty}$ increase as the temperature decreases. The work done on the surroundings depends linearly on these constants, thus leading to the observed increase in activation energy with decreasing temperature.

Actually, it is only the shear modulus that is important. To show this we refer to the theory of elasticity of isotropic media [58], assuming that the activation volume is relatively small. If $V$ is the volume of the cooperatively rearranging region, it is assumed that $\Delta V \ll V$. The activation energy is the elastic energy stored in the surroundings when the volume of the region has expanded to $V+\Delta V$. Elasticity theory concerns the relation between stress tensor, $\sigma_{i j}$, and strain tensor, $u_{i j}$. The latter is defined by

$u_{i j}=\frac{1}{2}\left(\partial_{i} u_{j}+\partial_{j} u_{i}\right)$,

where $\partial_{i} \equiv \partial / \partial x_{i}$ and $u_{i}$ is the $i$ th component of the elastic displacement vector, $\boldsymbol{u}$. For an isotropic solid the bulk and shear moduli $K$ and $G$ are defined [58] by (sum over repeated indices)

$\sigma_{i j}=K u_{l l} \delta_{i j}+2 G\left(u_{i j}-\frac{1}{3} \delta_{i j} u_{l l}\right)$.

The equation for static equilibrium is

$\partial_{i} \sigma_{i j}=0$.

Substituting Eq. (10) into Eq. (11) and subsequently Eq. (11) into Eq. (12) leads to

$\left(K+\frac{1}{3} G\right) \nabla(\nabla \cdot \boldsymbol{u})+G \nabla^{2} \boldsymbol{u}=\boldsymbol{0}$.

For a purely radial displacement $\nabla \times \boldsymbol{u}=\boldsymbol{0}$ and thus, via the well-known vector identity $\nabla \times(\nabla \times \boldsymbol{u})=\nabla(\nabla \cdot \boldsymbol{u})-\nabla^{2} \boldsymbol{u}$, we have $\nabla^{2} \boldsymbol{u}=$ $\nabla(\nabla \cdot \boldsymbol{u})$. When this is substituted into Eq. (13) one finds

$\nabla(\nabla \cdot \boldsymbol{u})=\boldsymbol{0}$,

implying that $\nabla \cdot \boldsymbol{u}=C_{1}$, where $C_{1}$ is a constant. The displacement (which is radial) is found by solving $\nabla \cdot \boldsymbol{u}=r^{-2} \partial_{r}\left(r^{2} u_{r}\right)=C_{1}$, leading to $u_{r}=C_{2} r^{-2}+C_{1} r / 3$. The latter term diverges as $r \rightarrow \infty$ and thus $C_{1}=0$. In conclusion $\nabla \cdot \boldsymbol{u}=0$, i.e., there is no compression of the surroundings during a flow even. Consequently the elastic energy is proportional to the shear modulus. 
If the radius of the region before the expansion is $R$ and the change of radius is $\Delta R$, we have since $\Delta R \ll R$

$u_{r}=\Delta R \frac{R^{2}}{r^{2}}(r>R)$.

The energy density of an elastic solid [58] is $(1 / 2) K u_{l l}^{2}+G\left(u_{i j}-(1 / 3) \delta_{i j} u_{l l}\right)^{2}$. Since $u_{l l}=0$ the energy density is given by $G u_{i j} u_{i j}=G\left(u_{r r}^{2}\right.$ $+u_{\phi \phi}^{2}+u_{\theta \theta}^{2}$ ) (all mixed terms such as $u_{r \phi}^{2}$ are zero because the displacement is purely radial). When Eq. (15) is used in the definition of the strain tensor in polar coordinates, we get for the energy density $6 G(\Delta R)^{2} R^{4} r^{-6}$. Thus, the elastic energy is given by

$\int_{R}^{\infty} 6 G(\Delta R)^{2} R^{4} r^{-6}\left(4 \pi r^{2}\right) \mathrm{d} r=8 \pi G(\Delta R)^{2} R$.

Substituting $V=4 \pi R^{3} / 3$ and $\Delta V=4 \pi R^{2} \Delta R$ into Eq. (16) we find, introducing the "characteristic volume"

$V_{\mathrm{c}}=\frac{2}{3} \frac{(\Delta V)^{2}}{V}$,

for the activation energy (with $G \equiv G_{\infty}(T)$ )

$\Delta E(T)=G_{\infty}(T) V_{\mathrm{c}}$.

For the average relaxation time we thus have [36]

$\tau=\tau_{0} \exp \left[\frac{G_{\infty}(T) V_{\mathrm{c}}}{k_{\mathrm{B}} T}\right]$.

Interestingly, extended mode-coupling theory leads to an expression resembling Eq. (19), except that $G_{\infty}$ is replaced by the zero-frequency bulk modulus [59]. The prediction of Eq. (19) was compared to experiments on a number of organic liquids in Ref. [36], assuming that $V_{\mathrm{c}}$ is temperature-independent. Strictly speaking, this assumption is inconsistent with the energy minimization leading to Eq. (9), but for a strongly anharmonic potential it is easy to show that the temperature dependence of $V_{\mathrm{c}}$ is negligible compared to that of $G_{\infty}$. In Ref. [36] the following version of the "Angell plot" [26] was used: instead of plotting the logarithm of viscosity as function of $T_{\mathrm{g}} / T$, it was plotted as function of $x=G_{\infty}(T) / T$ normalized to one at $T_{\mathrm{g}}$. The model predicts that a straight line should result, pointing to a physically reasonable viscosity prefactor at $x=0$. Ref. [36] compared this prediction to measurements done by three different methods. Overall, good agreement with the model prediction was found.

\section{Conclusions}

Early phenomenological models link the nonArrhenius average relaxation time of viscous liquids to configurational entropy, free volume, or potential energy; in Section 2 critiques of these models were summarized. The shoving model presents an alternative approach to the non-Arrhenius problem by relating $\tau(T)$ to the instantaneous shear modulus. The starting point of the model is the fact that intermolecular forces are anharmonic. Anharmonicity enters the model at three stages. First, the strong repulsions imply that it is very costly for molecules to rearrange at constant volume (a qualitative argument reminiscent of the free volume model). Secondly, anharmonicity implies that the shoving work greatly exceeds the "inner" energy barrier, a quantitative argument). Finally, the fact that $G_{\infty}$ depends on temperature is itself a consequence of anharmonicity (a perfectly harmonic solid has no temperature-dependence of its elastic constants). Note that according to the model a liquid is expected to be more fragile the more anharmonic it is. This trend is what Angell conjectured arguing within the entropy model [10].

The shoving model basically involves three postulates: (1) The main contribution to the activation energy is elastic energy; (2) This elastic energy is located in the surroundings of the reorienting molecules; (3) The elastic energy is shear energy. It is interesting to note that the model of Bueche [60] also links $\tau(T)$ to the elastic properties of the surroundings: A particular molecule is regarded as surrounded by spherical shells of molecules, shells that are bound elastically to each other. Bueche's idea is that if all concentric shells should vibrate outward in phase, the innermost shell would expand greatly, leaving the central molecule in a rather large hole so it could move to a new 
position. To calculate the probability of this happening Bueche made some further assumptions, leading to an expression that at high temperatures gives an Arrhenius expression, but at low temperatures a VFT-expression.

Returning to the shoving model, even if one basically accepts the above three postulates, there are a number of points leading potentially to deviations from Eq. (19): (1) Eq. (19) is based on a continuum approximation that may not be applicable at the molecular level; (2) The "inner" contribution to the activation energy has been ignored; (3) In real flow events spherical symmetry is probably violated, leading to some compression of the surroundings and thus a contribution to the activation energy proportional to $K_{\infty}$.

Ref. [36] gave a discussion of models related to the shoving model. To the best of the author's knowledge, the first to predict an expression equivalent to Eq. (19) was Nemilov [61] who in 1968 arguing quite differently - arrived at Eq. (19) with our $V_{\mathrm{c}}$ identified with the total region volume. At the present meeting Buchenau presented a model also leading to Eq. (19) for the average relaxation time [62]. As emphasized by Buchenau both here and previously [63], models of this type link short time dynamics to long time dynamics. At first sight such a link may seem surprising, but it makes sense because the transition itself is a very fast process [64]. Finally, we note [65] that the present mechanism may possibly be applied also to explain the non-Arrhenius average relaxation times of plastic crystals [66] and orientational glasses [67].

\section{Acknowledgements}

The author wishes to thank Professor G. Williams for drawing attention to the work of F. Bueche. This work was supported by the Danish Natural Science Research Council.

\section{References}

[1] W. Kauzmann, Chem. Rev. 43 (1948) 219.

[2] G. Harrison, The Dynamic Properties of Supercooled Liquids, Academic Press, New York, 1976.
[3] S. Brawer, Relaxation in Viscous Liquids and Glasses, American Ceramic Society, Columbus, OH, 1985.

[4] G.P. Johari, in: K.L. Ngai, G.B. Wright (Eds.), Relaxations in Complex Systems, US Government Printing Office, Washington, DC, 1985, p. 17.

[5] J. Jäckle, Rep. Progr. Phys. 49 (1986) 171.

[6] C.A. Angell, J. Phys. Chem. Solids 49 (1988) 863.

[7] G.W. Scherer, J. Non-Cryst. Solids 123 (1990) 75.

[8] C.A. Angell, J. Non-Cryst. Solids 131-133 (1991) 13.

[9] A. Hunt, J. Non-Cryst. Solids 160 (1993) 183.

[10] C.A. Angell, Science 267 (1995) 1924.

[11] U. Mohanty, Adv. Chem. Phys. 89 (1995) 89.

[12] M.D. Ediger, C.A. Angell, S.R. Nagel, J. Phys. Chem. 100 (1996) 13200.

[13] G. Adam, J.H. Gibbs, J. Chem. Phys. 43 (1965) 139.

[14] G.S. Grest, M.H. Cohen, Adv. Chem. Phys. 48 (1981) 455.

[15] M. Goldstein, J. Chem. Phys. 51 (1969) 3728.

[16] S.A. Brawer, J. Chem. Phys. 81 (1984) 954.

[17] M. Goldstein, Faraday Symp. Chem. Soc. 6 (1972) 7.

[18] G. Williams, in: M. Davies (Ed.), Dielectric and Related Molecular Processes, Specialist Periodical Report, vol. 2, Chem. Soc., London, 1975, p. 151.

[19] E. Donth, Glasübergang, Akademie Verlag, Berlin, 1981.

[20] E. Donth, J. Non-Cryst. Solids 53 (1982) 325.

[21] J.C. Dyre, Phys. Rev. Lett. 58 (1987) 792.

[22] F.H. Stillinger, J. Chem. Phys. 89 (1988) 6461.

[23] R.V. Chamberlin, Phys. Rev. B 48 (1993) 15638.

[24] U. Mohanty, I. Oppenheim, C.H. Taubes, Science 266 (1994) 425.

[25] J.C. Dyre, Phys. Rev. B 51 (1995) 12276.

[26] C.A. Angell, in: K.L. Ngai, G.B. Wright (Eds.), Relaxations in Complex Systems, US Government Printing Office, Washington, DC, 1985, p. 3.

[27] R. Böhmer, K.L. Ngai, C.A. Angell, D.J. Plazek, J. Chem. Phys. 99 (1993) 4201.

[28] R. Böhmer, J. Non-Cryst. Solids 172-174 (1994) 628.

[29] G.P. Johari, Ann. NY Acad. Sci. 279 (1976) 117.

[30] L.M. Torell, L. Börjesson, M. Elmroth, J. Phys.: Condens. Matter 2 (1990) 207.

[31] D. Kivelson, G. Tarjus, X. Zhao, S.A. Kivelson, Phys. Rev. E 53 (1996) 751.

[32] M.H. Cohen, D. Turnbull, J. Chem. Phys. 31 (1959) 1164.

[33] D. Turnbull, M.H. Cohen, J. Chem. Phys. 34 (1961) 120.

[34] D. Turnbull, M.H. Cohen, J. Chem. Phys. 52 (1970) 3038.

[35] J.H. Gibbs, E.A. DiMarzio, J. Chem. Phys. 28 (1958) 373.

[36] J.C. Dyre, N.B. Olsen, T. Christensen, Phys. Rev. B 53 (1996) 2171.

[37] J.C. Dyre, N.B. Olsen, T. Christensen, in: M. Giordano, D. Leporini, M.P. Tosi (Eds.), Non-Equilibrium Phenomena in Supercooled Fluids, Glasses and Amorphous Materials, Series in Contemporary Chemical Physics, vol. 12, 1996, p. 375.

[38] W.E. Roseveare, R.E. Powell, H. Eyring, J. Appl. Phys. 12 (1941) 669.

[39] A.K. Doolittle, J. Appl. Phys. 22 (1951) 1471.

[40] A.J. Barlow, J. Lamb, A.J. Matheson, Proc. R. Soc. London, Ser. A 292 (1966) 322. 
[41] H. Tweer, J.H. Simmons, P.B. Macedo, J. Chem. Phys. 54 (1971) 1952.

[42] W.T. Laughlin, D.R. Uhlmann, J. Phys. Chem. 76 (1972) 2317.

[43] F. Stickel, E.W. Fischer, R. Richert, J. Chem. Phys. 102 (1995) 6251.

[44] R.O. Davies, G.O. Jones, Proc. Roy. Soc. A 217 (1953) 26.

[45] M. Goldstein, J. Chem. Phys. 39 (1963) 3369.

[46] C.A. Angell, W. Sichina, Ann. NY Acad. Sci. 279 (1976) 53.

[47] Th.M. Nieuwenhuizen, Phys. Rev. Lett. 79 (1997) 1317.

[48] C.A. Angell, J.C. Tucker, J. Phys. Chem. 78 (1974) 278.

[49] C.A. Angell, J. Non-Cryst. Solids 102 (1988) 205.

[50] C.A. Angell, K.J. Rao, J. Chem. Phys. 57 (1972) 470.

[51] M. Goldstein, J. Chem. Phys. 64 (1976) 4767.

[52] P.D. Gujrati, M. Goldstein, J. Chem. Phys. 74 (1981) 2596.

[53] E.A. DiMarzio, A.J.M. Yang, J. Res. Natl. Inst. Stand. Technol. 102 (1997) 135.

[54] H. Bässler, Phys. Rev. Lett. 58 (1987) 767.

[55] V.I. Arkhipov, H. Bässler, D.V. Khramtchenkov, J. Phys. Chem. 100 (1996) 5118.
[56] B. Widom, Science 157 (1967) 375.

[57] D. Chandler, J.D. Weeks, H.C. Andersen, Science 220 (1983) 787.

[58] L.D. Landau, E.M. Lifshitz, Theory of Elasticity, Pergamon, Oxford, 1970.

[59] L. Sjögren, Z. Phys. B 79 (1990) 5.

[60] F. Bueche, J. Chem. Phys. 30 (1959) 748.

[61] S.V. Nemilov, Russ. J. Phys. Chem. 42 (1968) 726.

[62] U. Buchenau, presented at 3rd Int. Discussion Workshop on Relaxations in Complex Systems, Vigo, Spain, 30 June 11 July 1997.

[63] U. Buchenau, R. Zorn, Europhys. Lett. 18 (1992) 523.

[64] R.W. Hall, P.G. Wolynes, J. Chem. Phys. 86 (1987) 2943.

[65] M. Goldstein, in: K.L. Ngai, G.B. Wright (Eds.), Relaxations in Complex Systems, US Government Printing Office, Washington, DC, 1985, p. 13.

[66] H. Suga, S. Seki, J. Non-Cryst. Solids 16 (1974) 171.

[67] U.T. Höchli, K. Knorr, A. Loidl, Adv. Phys. 39 (1990) 405. 